\title{
ROBUST BOOLEAN SET OPERATION BY SYMBOL PROCESSING OF TOPOLOGY
}

\author{
Masatake Higashi, Satoshi Torii, Mamoru Hosaka \\ Toyota Technological Institute \\ 2-12-1, Hisakata, Tempaku-ku, Nagoya 468-8511, Japan \\ higashi@toyota-ti.ac.jp
}

Hiroyuki Nakamura

Nihon Unisys Ltd.

1-1-1, Toyosu, Koto-ku, Tokyo 135-8560, Japan

\begin{abstract}
This paper describes a robust method for the Boolean set operations for solid models. The method always produces a consistent solid model regardless of numericla errors, by employing symbol processing algorithms which satisfy its topological constraints. The degeneracies of the solids are obtained according to the ambiguity caused by the given tolefances and numerical errors, which decide the resolution of calculation for the set operation.
\end{abstract}

Keywords: Set operation, symbol processing, topology, robustness, tolerance

\section{INTRODUCTION}

In recent years, solid modeling systems including parametric modeling functions, as the central components of CAD/CAM systems to establish digital processes through the product life cycle, have been widely applied to the design and manufacturing of mechanical parts or industrial products. However, there still remain serious problems in the current solid modeling systems concerning robustness and flexibility. Therefore, we carry out this research with the aim of establishing a robust method for the Boolean set operations for solid models, that is a method which can always produce a solid object which is valid within the prescribed precision regardless of numerical errors. In order to achieve this goal, we perform the following studies. 
1 We generate only valid solid objects without failures, satisfying the topological consistency conditions by means of symbol processing algorithms which automatically satisfy the topological constraints.

2 We maintain the overall topological consistency of the solid by symbolically detecting those local regions where topological inconsistency is caused by numerical errors of geometric calculations, and modifying them as ambiguous regions.

3 We extend these methods for polyhedral solids to non-manifold solids and curved solids.

Various approaches have been made towards robustness of the set operations. The approaches are classified in two categories: one using exact arithmetic and the other using floating point arithmetic. The approaches in the first category use either variable length multiprecision rational numbers(Beno93) or finite precision exact numbers(BanRos96; Fort95; SugiIri89). In these approaches, one needs not worry about numerical errors. But these approaches are confined to polyhedral solids since many geometric algorithms for curved solids depend on iterative approximation methods.

In the approaches in the second category, each geometric calculation is accompanied by numerical errors, but one must construct a topological structure for the output solid based on these geometric calculations. In order to do this construction, tolerances are generally used for the incidence tests between geometric entities. However, using a fixed tolerance value, the results of different incidence tests may contradict each other(AgrReq94; Zhu93). Approaches solving this difficult problem include those using adaptive or local tolerances(Jack95; Seg90), and those using symbolic reasoning(Hoff89). In both approaches, the algorithm gets rather complicated while inconsistencies are not completely avoided. In contrast to these approaches which use tolerances for the incidence tests, Sugihara(Sugi94) proposes a completely robust and consistent algorithm, which uses no tolerances and does no incidence tests, for the intersection of convex polyhedra. This approach, which puts emphasis on topological consistency and treats numerical values secondarily, seems promising for the development of robust geometric algorithms. But currently, its application to the set operation is limited to the intersection of convex polyhedra, while applications to the construction of Voronoi diagrams(SugiIri92) are successful.

We take a similar approach to Sugihara's one, but we extend it to all the kinds of set operations for any type of solids. We use tolerances for obtaining degenerate states of input solids such as coincidences of two faces, an edge and a face and so on, which are rather preferable for industrial users because they regard such ambiguous states by the numerical errors as coincident ones.

First, we have developed a method(Naka97) for the construction of the intersection graph between two polyhedral solids, which is the first stage of the Boolean operation. The method, in order not to be affected by numerical errors, determines the connection of the intersection lines based on the topological connectivity properties of the edge-face intersection points represented symbol- 
ically by face names. The method employs a new algorithm for the detection of the degeneracy such as vertex coincidence and edge crossing, by constructing clusters of the intersection points which represent the set of intersection points not distinguishable numerically and topologically. We construct the intersection graph based on the connectivity between the clusters and the intersection line segments.

Second, we have presented a method(Naka98) for the construction of the topological structure of the output solid - the latter part of the Boolean operation. Here, using the face-based representation(Higa95) as a basis for the topological structure of a polyhedral solid, instead of the conventional pointerbased structures for the boundary representations, we have introduced a new structure which explicitly holds the topological constraints for the model. These topological constraints represent the coincidence or degeneracy detected during the Boolean operation along with those intended by the designer in a uniform manner as constraints between the faces of the solid. By this structure, we are able to avoid the expansion of inconsistency in solid model data caused by accumulated numerical errors when Boolean operations are applied iteratively, and are also able to represent the intentions of the designer within a solid model. At the same time, this solid structure represents non-manifold solids uniformly with manifold solids, and forms a category of polyhedral solids closed under Boolean operations.

Third, we have developed a method which generates a valid solid model when numerical errors exceed the designated tolerances. This is a main theme of this paper. We detect the inconsistency of topology caused by the numerical errors, by finding out an unclosed part of the intersection graph and a contradictory coincidence relation. We then modify the topological structure so as to treat the concerned part as an ambiguous region by changing the tolerances, while the global topological structure remain the same. This generates a consistent topological structure according to the designated precision, which is partly made larger by the numerical errors.

In this paper, we describe concepts of set operation by symbol processing in Section 2. Then, we propose robust algorithms against numerical errors in Section 3. After presenting their application examples in Section 4, we summarize the paper.

\section{SET OPERATION BY SYMBOL PROCESSING}

\subsection{Generation of intersectin graph}

An intersection graph is a graph whose edges are the valid intersection line segments between two input solids, and whose vertices are the endpoints of these segments. Here, valid intersection line segments are those parts of the intersection lines between face planes (the infinite plane on which the face lies) of two solids, which lie on the valid regions of both faces. 
The construction of the intersection graph is performed in the following steps:

1 Calculate all edge-face intersection points and associate a symbolical representation to each intersection point.

2 Make clusters of the intersection points based on their symbolical connectivity.

3 Extract valid intersection line segments based on the symbolical connectivity of the clusters.

We first calculate the intersection points between edges and face planes of the two solids, and associate each intersection point a triplet of face names as its "symbol", which represents the connectivity property of the intersection point.

When an edge $e$ of solid $S_{1}$ intersects a face plane $g$ of solid $S_{2}$, and the two side faces of $e$ are $f_{1}$ and $f_{2}$, then the symbol of the intersection point is defined as follows:

- First, define the direction of edge e so that the face $f_{1}$ is to the right as seen from outside of $S_{1}$.

- When e penetrates $g$ from inside to outside (of $S_{2}$ ), then the symbol is $\left(g, f_{1}, f_{2}\right)$.

- When e penetrates $g$ from outside to inside, then the symbol is $\left(g, f_{2}, f_{1}\right)$.

The symbol of the intersection point represents the "connectivity" in the following sense. When we define the direction of the intersection lines so that the faces of solid $A$ are on the left (and the faces of solid $B$ on the right) on the intersection solid $A \cap B$, these directed intersection lines are represented by pairs of face names $(f, g)$, where $f$ is a face of $A$ and $g$ is a face of $B$. Then, we can represent symbolically which intersection line "comes into" an intersection point and which "goes out" as follows:

$$
\begin{aligned}
& \stackrel{(1, a)}{\longrightarrow}(1, a, b) \stackrel{(1, b)}{\longrightarrow} \\
& \stackrel{(2, a)}{\longrightarrow}(a, 1,2) \stackrel{(1, a)}{\longrightarrow}
\end{aligned}
$$

where the faces of solid $A$ are denoted by numbers and faces of $B$ by alphabets. Whether an intersection line "comes into" or "goes out" is determined by whether or not the ordering of the face names is the same as that in the symbol of the intersection pont.

In degenerate cases such as vertex-vertex coincident or edge-crossing cases, multiple edge-face intersection points exist in a very small region. In these cases, the determination of valid intersection segments and their connections becomes a numerically subtle problem. For example, in an almost edge-crossing case (Fig. 1), a small numerical error may lead to a wrong decision on the 


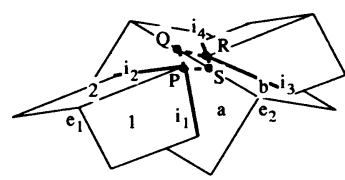

(a) Consistent case 1

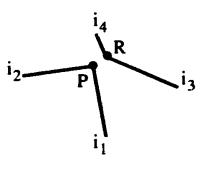

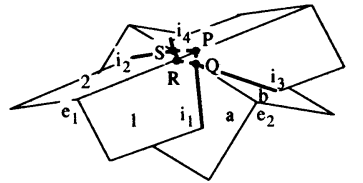

(b) Consistent case 2

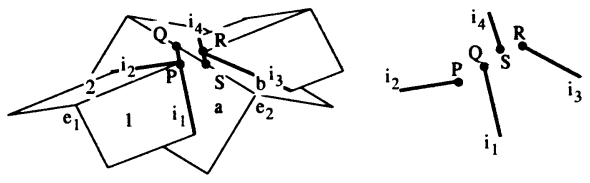

(c) Inconsistent case

Figure 1 Connection of intersection lines in nearly edge-crossing cases

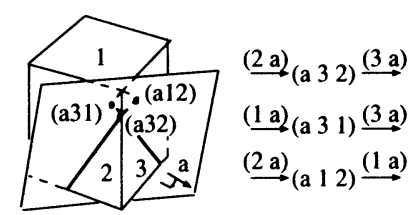

(a) Vertex-face coincident case: $\{($ a 3 2), (a 3 1), (a 12$)\}$

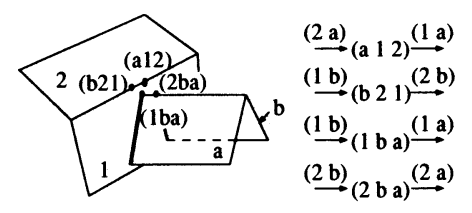

(b) Edge-crossing case:

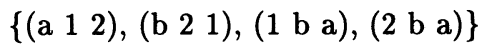

Figure 2 Clusters corresponding to degeneracy

ordering of edge-face intersection points on the intersection lines, and then the intersection segments cannot be connected correctly as illustrated in Fig. 1 (c).

In order not to be influenced by numerical errors, we should detect the degeneracy by a non-numerical method, or at least utilize non-numerical information in the detection of degeneracy. Therefore, we exploit the symbolical connectivity relations of the intersection points to make the "clusters", namely the sets of intersection points which are not distinguishable topologically or numerically, and should be considered as identical.

The clusters are constructed by the following algorithm:

When the symbols of two edge-face intersection points have the same input or output, and these points are nearer than the prescribed tolerance, we put them in a same cluster.

By this algorithm, the multiple edge-face intersection points corresponding to degeneracy are made into clusters (Fig 2).

In order to construct the intersection graph, we must extract the valid segments out of the infinite intersection lines between the face planes of two solid 


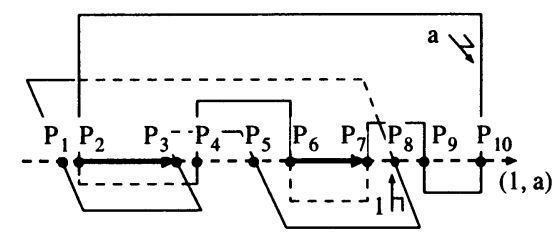

Figure 3 Extraction of valid segments

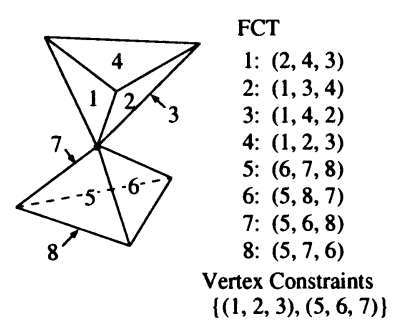

(a)

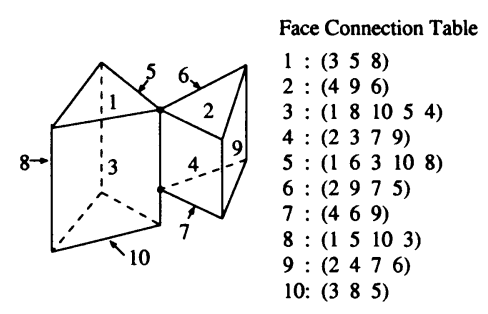

(b)

Figure 4 Data structure for polyhedra

(Fig 3). Using the connectivity properties of the clusters which are derived by those of the intersection points, this extraction can be performed completely non-numerically as presented in (Naka97).

\subsection{Generation of topological structure}

We adopt a face-based data representation augmented by the constraint conditions which represent the degeneracy detected in the Boolean operations or intended by the designer, following the idea which looks upon the faces as the most fundamental among the various entities constituting a solid model (Higa95). By adopting this structure, we can avoid the expansion of inconsistency by accumulation of numerical errors, at the same time gaining flexibility to the designer's intension.

The topological structure of the output solid is constructed by making face loops by a symbolical procedure similar to the process desribed in the previous subsection. This method is simpler than the usual method which cuts the surfaces of input solids along the intersection and then sews together the appropriate parts to make the output solid, and more robust against numerical errors.

The polyhedral solids, which are the inputs and outputs of our set operation algorithm, are represented by the following structure:

1 Face connection table, 
2 Geometric data (equation coefficents) for each face plane,

3 Topological constraints.

The face connection table is defined as the set of all adjacent face loops of all faces in the solid. Here, an adjacent face loop is a face boundary loop represented as a list of face names. Topological constraints represent the degeneracy in the solid, and are constituted by the following three lists:

- Vertex constraints - list of face sets consisting of four or more faces and sharing a same vertex,

- Edge constraints - list of face sets consisting of three or more faces and sharing a same edge,

- Face constraints - list of face sets consisting of two or more faces which are coincident. (with same or opposite directions)

This structure has the ability to represent the non-manifold solids such as shown those in Fig. 4.

The topological structure for the output solid of Boolean operation is constructed by the following steps:

1 Generate the set of clusters and vertices which are to become the vertices of the output solid, by determining the IN-OUT of each vertices of each solid against the other solid.

2 Generate the set of coaxial faces, which are the set of three or more faces sharing a same line.

3 Extract the face set for the output solid from the set of clusters constructed in step 1 , and make the face loops for each face by connecting the relevant clusters based on their connectivity.

4 Generate the topological constraints for the output solid from the clusters and coaxial face sets.

For example, when we calculate the intersection $A \cap B$ of two cubes $A$ and $B$ shown in Fig. 5 (a), the edge-face intersection points and those vertices which lie inside of the other solid are the vertices for $A \cap B$ shown in Fig. (b). We make the face loops based on symbolical connectivity as shown in Fig. (c), and obtain the Face connection table for $A \cap B$ shown in Fig. (d).

\section{ROBUST ALGORITHMS AGAINST NUMERICAL ERRORS}

\subsection{Intersection graph for degeneracy}

The intersection graph on the occurrence of degeneracy is unified into one from those obtained by perturbing one of the input solids by a small distance. 


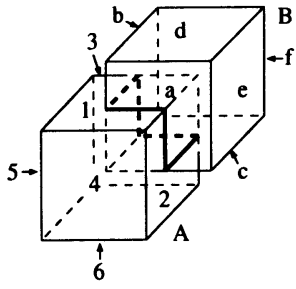

(a) Solids $A$ and $B$

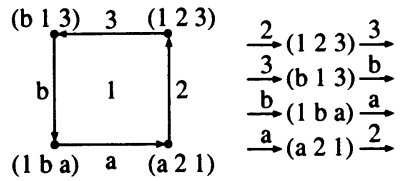

(c) Loop of face 1 in $A \cap B$

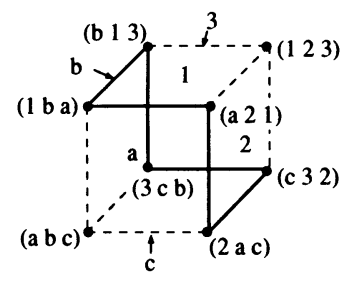

(b) Vertices and intersection points for $A \cap B$

1: $2,3, \mathrm{~b}, \mathrm{a}$

2: $1, \mathrm{a}, \mathrm{c}, 3$

3: $1,2, c, b$

a: $1, b, c, 2$

b: $1,3, \mathrm{c}, \mathrm{a}$

c: a, b, 3, 2

(d) FCT(Face Connection Table)

of $A \cap B$

Figure 5 Set operation by symbol processing

Namely, it represents multiple states. Fig. 6 shows an example for the case of edge-crossing. One intersection point has four edges joining two normal states here, although an intersection graph usually consists of intersection points with two arcs: coming in and going out edges. The graph is made automatically by connecting the clusters some of which consist of four symbols of the intersection points. Next, we show the case of face coincidence using Fig. 7. The graph is similarly unified from two states. Some nodes of the intersection graph have three edges here, because there exist singular intersection points, which have odd-number edges, such as those on face-face coincidence and face-edge coincidence. In these cases, intersection lines lie upon existing faces or edges and disappear there. But since there remain intersection point symbols on the coincident faces, we can connect them as intersection segments.

Based-on above discussion, we introduce the condition of the intersection graph to be consistent.

The intersection graph must consist of one or multiple loops which share some segments in the singular cases. 


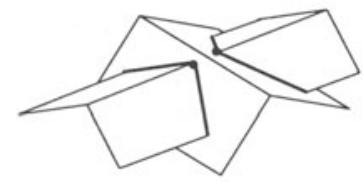

(a) Non-degenerate state 1

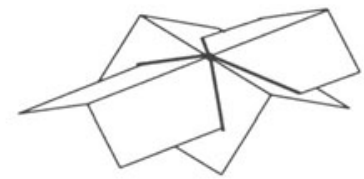

(b) Degenerate state

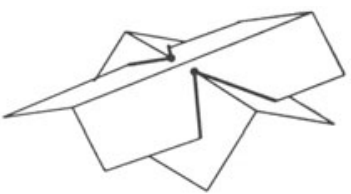

(c) Non-degenerate state 2

Figure 6 Multiple states for edge-crossing

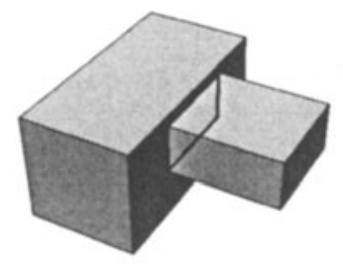

(a) Non-degenerate state 1

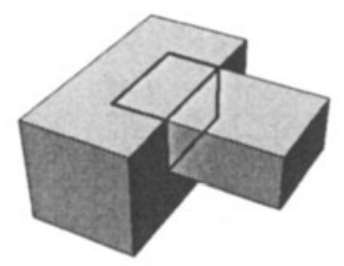

(b) Degenerate state

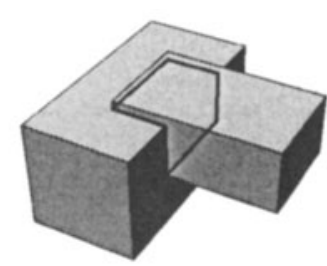

(c) Non-degenerate state 2

Figure 7 Multiple states for face coincidence

\subsection{Numerical errors and tolerance}

We use two types of tolerance in this research. One is an on-tolerance for the vertex-face coincidence, and the other is a cluster tolerance for unifying intersection points. The on-tolerance makes a vertex coincident to a face to obtain an intersection point certainly for the opposite position of the vertex against the face because of the numerical errors, when the distance from the vertex to the face is within the tolerance range. For the case that both end points of an edge are on a face, the edge is regarded to be coincident with the face. On the other hand, the cluster tolerance obtains valid intersection segments inside the face boundary by combining intersection points reversely ordered along the intersection due to numerical errors.

In the above judgment, we suppose that the numerical errors are smaller than the tolerances. Now, we discuss the relation between the numerical errors and the tolerances for the consistency of the intersection graph. When the errors are larger than the on-tolerance, intersection lines are possible to be missed, added by mistake, or connected to false edges according to how the incident edges to the vertex cross the face, but the intersection graph is kept consistent corresponding to the given results. However, when the errors are larger than the cluster tolerance, the intersection loop is open because intersection point symbols determined using wrong valid segments cannot be connected. If one changes the tolerance value larger to avoid the above cases, he or she can con- 
nect the concerned part of the graph, but more false intersection points are included which are for example those outside the face boundary. We should select appropriate intersection points so as to make a consistent topology instead of selecting a suitable tolerance value, because the larger tolerance cannot satisfy all the necessary conditions.

\subsection{Removal of inconsistency}

We describe algorithms to detect inconsistency of the intersection graph and to correct it.

When intersection segments have open ends, they are deleted until they reach the existing loops based on the judgment that they are false additional intersections. However, when an intersection point supposed to be reliable is to be deleted in the above procedure, we should not delete it.

The reliable intersection point is determined by the fact that it is not within the on-tolerance, that is, its edge surely intersects with the object face.

To make a consistent intersection graph for this case, we have to close the open ends of the intersection segments. By making the cluster tolerance larger, remaking and connecting the clusters again, we can connect them because they are open by the wrong decision for the valid segments.

Next, we consider the case of the contradiction for the coincidence among faces and edges. The coincidence is determined from the following facts. When both vertices of an edge are on a face, the edge is considered to be coincident with the face and a relation of co-axial of three faces is made. When a co-axial relation among four faces exists, two edges are coincident. Further, when a face has more than two pairs of co-axial relations with another face, the faces are coincident. However, when an edge intersects with a face with a very thin angle, there happens a contradiction such that its one vertex is on the face and the other vertex is out of the face, but the intermediate intersection point made by edge-crossing with another edge is set to "on" for the face. In this case, the edge should be changed to be on the face so as to be consistent for the degeneracy. When such cases occur side by side, a compound contradiction is made. To cope with these, the next algorithm is introduced.

Two clusters which share three faces are made to have a co-axial relation when they cannot be unified because of the topological constraints and the distance among the intersection points from each cluster is large enough, while they are made to be one cluster for the distance within the tolerance. To be consistent through the entire process, the vertices of the edges newly made co-axial are changed to be "on" the faces and the other coincidence relations are checked again.

We explain the above algorithm in Fig. 8 by the example shown in Fig. 7 . Two blocks are located so that two faces from each block are almost coincident. 


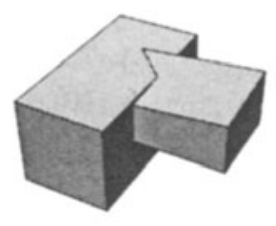

(a) $\varepsilon_{c}=10^{-6}$ (no degeneracy)

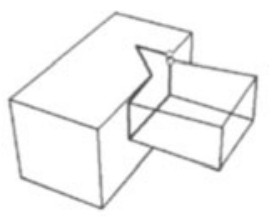

(b) Error is larger

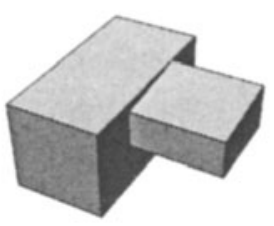

(c) $\varepsilon_{c}=10^{-5}$

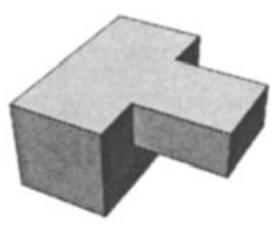

(d) $\varepsilon_{c}=10^{-4}$

Figure 8 Example of processing for consistency

If intersection calculations are strictly done without errors for the in-out judgment of intersection points against the boundaries of faces, the output solid is generated without degeneracy as shown in Fig. (a). When the numerical errors are larger than the tolerance, the intersection graph is not closed as shown in Fig. (b). So, the cluster tolerance is changed larger locally and automatically, and the output becomes to have some degeneracy. Instead of giving larger numerical errors, we set the cluster tolerance $\varepsilon_{c}$ larger. For $\varepsilon_{c}=10^{-5}$, one edge is changed to be incident with a face as shown in Fig. (c), and for a larger tolerance as $\varepsilon_{c}=10^{-4}$, face coincidence is generated as shown in Fig. (d). We get valid outputs which satisfy the given precision, that is resolution of the calculation, according to the tolerance values.

\section{APPLICATION EXAMPLE}

We apply the above algorithms to some examples. We implemented them on Mathematica (Wolfram Research Inc.) and later in Common Lisp. All numerical calculations are carried out in double precision floating point arithmetic.

First, we check the results of the set operation for two unit cubes, when one of them is rotated around $x, y, z$ axes by angle $\theta$. Fig. 9 shows input solids and an output of union in case of $\theta=30^{\circ}$. We change the angle from $1^{\circ}$ to $10^{-6 \circ}$ and vary the on-tolerance $\varepsilon$ and cluster tolerance $\varepsilon_{c}$ from $10^{-2}$ to $10^{-15}$. Table 1 shows some results for $\theta=0.001^{\circ}$. Here, numbers of intersection point symbols(S), clusters(C), intersection segments(I) and reduced intersection segments $(R)$ are described. By the numbers, we can classify the patterns of outputs according to the degeneracy. Table 2 shows various output patterns for various values of $\theta$ and tolerances. For each $\theta$, certain value $\varepsilon^{p}$ (left column) exists, and when $\varepsilon$ is larger than or equal to that, two solids cannot be discriminated. Output patterns are classified by values of $\varepsilon_{c}$ into local coincidence, Pattern 1 and Pattern 2. Two main patterns are shown in Fig. 10 with intersection graphs. Pattern 1 has 8 clusters all made of multiple intersection segments, and Pattern 2 has four clusters of edge-crossing and 12 normal intersection points which is the same to the case of $\theta=30^{\circ}$. When $\varepsilon$ is smaller than $\varepsilon^{p}$ and larger than or equal to cartain value $\varepsilon^{q}$ (right column), 


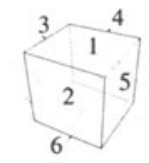

(a) Cube A

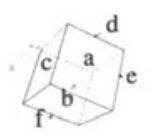

(b) Cube B

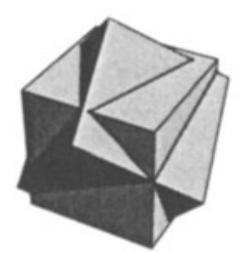

(c) Intersecting cubes

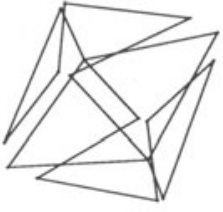

(d) Intersection lines

Figure 9 Output solid for $\theta=30^{\circ}$

Table 1 Outputs of various tolerance values $\left(\theta=0.001^{\circ}\right)$

\begin{tabular}{|c|c|c|c|c|c|c|c|c|}
\hline$\varepsilon$ & & $1.0 \mathrm{E}-2$ & $1.0 \mathrm{E}-5$ & $1.0 \mathrm{E}-6$ & $1.0 \mathrm{E}-10$ & $1.0 \mathrm{E}-12$ & $1.0 \mathrm{E}-13$ & $1.0 \mathrm{E}-14$ \\
$\varepsilon$ & & $\ldots 1.0 \mathrm{E}-4$ & & $\ldots 1.0 \mathrm{E}-9$ & $\ldots 1.0 \mathrm{E}-11$ & & & $\ldots .0 \mathrm{E}-15$ \\
\hline 0.01 & $\mathrm{~S}$ & 48 & 48 & 48 & 48 & 48 & 48 & 48 \\
\cline { 2 - 9 }$\ldots$ & $\mathrm{C}$ & 8 & 8 & 8 & 8 & 8 & 8 & 8 \\
\cline { 2 - 9 } $1.0 \mathrm{E}-4$ & $\mathrm{I}$ & 24 & 24 & 24 & 24 & 24 & 24 & 24 \\
\cline { 2 - 9 } & $\mathrm{R}$ & 24 & 24 & 24 & 24 & 24 & 24 & 24 \\
\hline \multirow{2}{*}{$1.0 \mathrm{E}-5$} & $\mathrm{~S}$ & $54^{*}$ & $72^{*}$ & 84 & 84 & 84 & 84 & 84 \\
\cline { 2 - 9 }$\ldots$ & $\mathrm{C}$ & 8 & 8 & 8 & 16 & 16 & 16 & 16 \\
\cline { 2 - 9 } $1.0 \mathrm{E}-9$ & $\mathrm{I}$ & 21 & 18 & 24 & 24 & 24 & 24 & 24 \\
\cline { 2 - 9 } & $\mathrm{R}$ & 21 & 16 & 18 & 18 & 18 & 18 & 18 \\
\hline \multirow{3}{*}{$1.0 \mathrm{E}-10$} & $\mathrm{~S}$ & 48 & 48 & 48 & 48 & 48 & 48 & 48 \\
\cline { 2 - 9 }$\ldots$ & $\mathrm{C}$ & 8 & 8 & 8 & 16 & 16 & 16 & 16 \\
\cline { 2 - 9 } & $\mathrm{I}$ & 18 & 18 & 18 & 18 & 18 & 18 & 18 \\
\cline { 2 - 9 } & $\mathrm{R}$ & 18 & 18 & 18 & 18 & 18 & 18 & 18 \\
\hline \multirow{3}{*}{$1.0 \mathrm{E}-15$} & $\mathrm{~S}$ & 44 & 44 & 44 & 44 & 44 & 44 & 44 \\
\cline { 2 - 9 } & $\mathrm{C}$ & 8 & 8 & 8 & 16 & 16 & 16 & 16 \\
\cline { 2 - 9 } & $\mathrm{I}$ & 18 & 18 & 18 & 18 & 18 & 18 & 18 \\
\cline { 2 - 9 } & $\mathrm{R}$ & 18 & 18 & 18 & 18 & 18 & 18 & 18 \\
\hline
\end{tabular}

S: Number of edge-face symbols(*: co-axial relations are modified), C: Number of clusters, I: Number of intersection segments, R: Number of reduced intersection segments

additional intrsection segments exist and they are deleted as open loops. At these $\varepsilon$ values, if $\varepsilon_{c}$ is larger than or equal to $\varepsilon_{c}^{p-1}$, local coincidence where some pairs of faces are coincident is output by modifying the inconsistency of topology adding co-axial relations. Further, when both tolerances are small for $\theta=10^{-4 \circ}$ and $\theta=10^{-5 \circ}$, Pattern 2 cannot be output because of open loops of intersection graphs, instead they are modified to Pattern 1 by enlarging $\varepsilon_{c}$. From the table, we understand that, for a larger tolerance, an output has more degeneracies and its resolution is getting worse to maintain its topological consistency. 


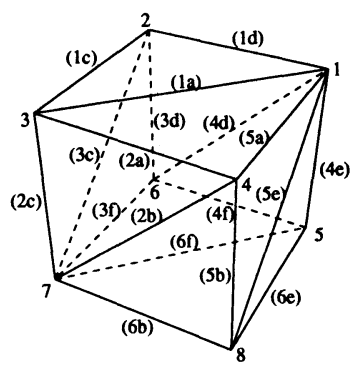

Pattern 1 (Intersection lines)

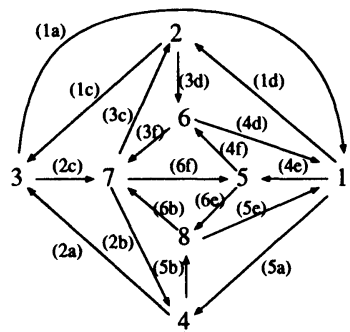

Pattern 1 (Cluster connections) Pattern 2 (Cluster connections)

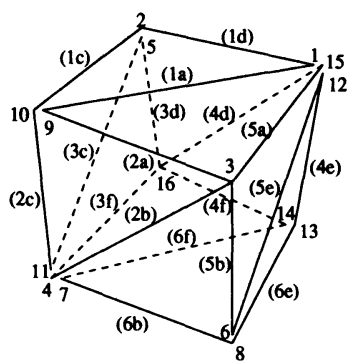

Pattern 2 (Intersection lines)

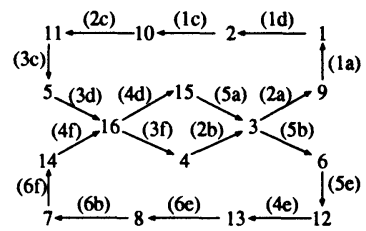

Figure 10 Output patterns of set operation for cubes

Table 2 Output patterns of various values of $\theta$ and tolerances

\begin{tabular}{|c|c|c|c|c|c|}
\hline$\theta$ & Coincidence & $\begin{array}{c}\text { Local } \\
\text { coincidence }\end{array}$ & Pattern 1 & Pattern 2 & $\begin{array}{c}\text { Additional } \\
\text { segments }\end{array}$ \\
\hline $1^{\circ}$ & $\varepsilon \geq 0.1$ & $\begin{array}{c}\left(\varepsilon \geq 10^{-3}\right) \\
\wedge\left(\varepsilon_{c} \geq 10^{-2}\right)\end{array}$ & $\varepsilon_{c} \geq 10^{-3}$ & $\varepsilon_{c} \leq 10^{-4}$ & $\varepsilon \geq 10^{-3}$ \\
\hline $0.1^{\circ}$ & $\varepsilon \geq 0.01$ & $\begin{array}{c}\left(\varepsilon \geq 10^{-5}\right) \\
\wedge\left(\varepsilon_{c} \geq 10^{-3}\right)\end{array}$ & $\varepsilon_{c} \geq 10^{-5}$ & $\varepsilon_{c} \leq 10^{-6}$ & $\varepsilon \geq 10^{-5}$ \\
\hline $0.01^{\circ}$ & $\varepsilon \geq 10^{-3}$ & $\begin{array}{c}\left(\varepsilon \geq 10^{-7}\right) \\
\wedge\left(\varepsilon_{c} \geq 10^{-4}\right)\end{array}$ & $\varepsilon_{c} \geq 10^{-7}$ & $\varepsilon_{c} \leq 10^{-8}$ & $\varepsilon \geq 10^{-7}$ \\
\hline $10^{-30}$ & $\varepsilon \geq 10^{-4}$ & $\begin{array}{c}\left(\varepsilon \geq 10^{-9}\right) \\
\wedge\left(\varepsilon_{c} \geq 10^{-5}\right)\end{array}$ & $\varepsilon_{c} \geq 10^{-9}$ & $\varepsilon_{c} \leq 10^{-10}$ & $\varepsilon \geq 10^{-9}$ \\
\hline $10^{-40}$ & $\varepsilon \geq 10^{-5}$ & $\begin{array}{c}\left(\varepsilon \geq 10^{-11}\right) \\
\wedge\left(\varepsilon_{c} \geq 10^{-6}\right)\end{array}$ & $\varepsilon_{c} \geq 10^{-11}$ & $\left(\varepsilon_{c} \leq 10^{-12}\right)^{*}$ & $\varepsilon \geq 10^{-11}$ \\
\hline $10^{-50}$ & $\varepsilon \geq 10^{-6}$ & $\begin{array}{c}\left(\varepsilon \geq 10^{-13}\right) \\
\wedge\left(\varepsilon_{c} \geq 10^{-7}\right)\end{array}$ & $\varepsilon_{c} \geq 10^{-13}$ & $\left(\varepsilon_{c} \leq 10^{-14}\right)^{*}$ & $\varepsilon \geq 10^{-13}$ \\
\hline $10^{-60}$ & $\varepsilon \geq 10^{-7}$ & $\begin{array}{c}\left(\varepsilon \geq 10^{-15}\right) \\
\left(\varepsilon_{c} \geq 10^{-8}\right)\end{array}$ & $\varepsilon_{c} \geq 10^{-15}$ & $-\cdots$ & $\varepsilon \geq 10^{-15}$ \\
\hline
\end{tabular}

$\dagger:$ co-axial relations are added

*: $\varepsilon_{c}$ is enlarged and inconsitent graph is changed into Pattern 1 


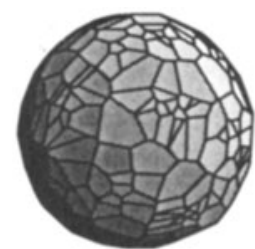

(a) 6th intersection

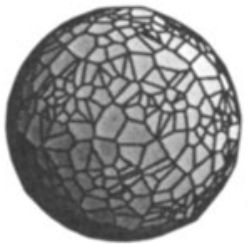

(b) 7 th intersection

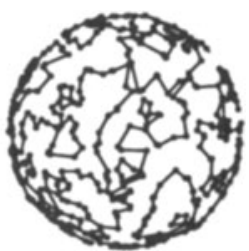

(c) 7th int.graph

Figure 11 Repeated intersection of solids

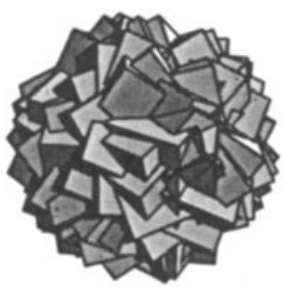

(d) 4th union

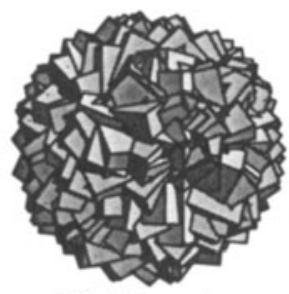

(e) 5th union

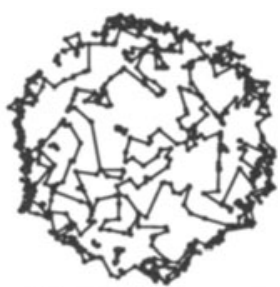

(f) 5 th int. graph

Figure 12 Repeated union of solids

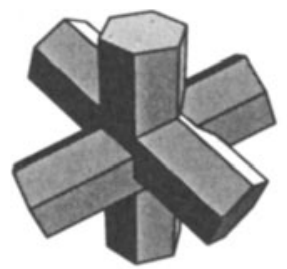

(a) Union

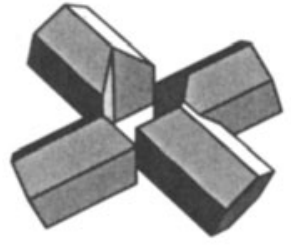

(b) Difference

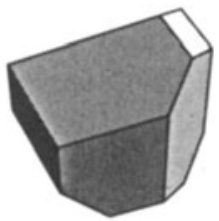

(c) Intersection

Figure 13 Face coincidence for six-sided prisms 
Next, we repeated above set operations each time with a random rotation angle to confirm the consistency against the accumulation of errors. Fig. 11 shows results of sixth and seventh intersections along with the intersection graph which has 1572 clusters for the seventh intersection. Fig. 12 shows results of fourth and fifth unions along with the intersection graph having 2778 clusters.

Last, we pick up examples for face-face coincidence. Three six-sided prisms are located in the position where four pairs of faces are coincident. The results are shown in Fig. 13. In Fig. (a) union, four pairs of faces are coincident in the same direction, and in Fig. (b) difference, in the opposite direction along with edge-edge coincidence in the non-manifold state.

Our method generates valid outputs according to the given resolution. This looks similar to the adaptive tolerance method, but our method is quite different from it on the point that our method generates only a valid solid topologically. On the other hand, the approach using exact arithmetic needs so much computation costs for calculating a too small range of intersections which are meaningless in the practical engineering. Nevertheless, it needs special treatments for coincidence calculations. Contrarily, the pure topology-oriented approach needs not such special treatments, but its outputs include mismatch of geometry and topology according to positional errors of the calculations, which sometimes exceed the resolution of the object.

\section{SUMMARY}

We have proposed algorithms for robust set operations even in the case that the numerical errors are larger than the given tolerance. Through the applied examples, we have obtained the following conclusion.

1 We obtain a valid output of a set operation without failure by symbol processing which generates an intersection graph according to the topological constraints.

2 The output solid varies corresponding to the tolerance and numerical errors which decide the resolution of the calculation.

Our future research is to extend this approach to curved solids. Part of it is now being developed. This research is partly supported by Grant-in-Aid for Scientific Research (A) (N0. 103558005) of the Ministry of Education, Science and Culture of Japan.

\section{References}

Agrawal, A. and Requicha, A.A.G. (1994). A Paradigm for the Robust Design of Algorithms for Geometric Modeling, Computer Graphics Forum (EUROGRAPHICS '94), 13 (3), pp.C32-C44.

Banerjee, R. and Rossignac, J.R. (1996). Topologically exact evaluation of polyhedra defined in CSG with loose primitives, Computer Graphics Forum, 15 (4), pp.205217. 
Benouamer, M., Michelucci, D., and Peroche, B. (1993). Error-Free Boundary Evaluation Using Lazy Rational Arithmetic; A Detailed Implementation, Proc. 2nd ACM Symposium on Solid Modeling, pp.115-125.

Fortune, S. (1995). Polyhedral modelling with exact arithmetic, Proc. 3rd ACM Symposium on Solid Modeling, pp.225-233.

Higashi, M., Torihara, F., Takeuchi, N., Sata, T., Saitoh, T. and Hosaka, M. (1995). Face-Based Data Structure and its Application to Robust Geometric Modeling, Proc. 3rd ACM Symposium on Solid Modeling, pp.235-246.

Hoffman, C.M., Hopcroft, J.E. and Karasick, M. (1989). Robust Set Operations on Polyhedral Solids, IEEE Computer Graphics and Applications, 9 (November), pp.50-59.

Jackson, D.J. (1995). Boundary Representation Modelling with Local Tolerances, Proc. 3rd ACM Symposium on Solid Modeling, pp.247-253.

Laidlaw, D.H. Trumbore, W.B. and Hughes, J.F. (1986). Constructive Solid Geometry for Polyhedral Objects, Computer Graphics (Proc. SIGGRAPH'86), 20 (4), pp.161-170.

Mäntylä, M. An Introduction to Solid Modeling, (1988). Computer Science Press.

Nakamura, H., Higashi, M., and Hosaka, M. (1997). Robust Computation of Intersection Graph between Two Solids, Computer Graphics Forum (EUROGRAPHICS '97), 16 (3), pp.C79-C88.

Nakamura, H. (1998) Robust Interference Computation for Solid Models Based on Symbol Processing, Doctor Thesis, Toyota Technological Institute.

Segal, M. (1990). Using Tolerances to Guarantee Valid Polyhedral Modeling Results, Computer Graphics (Proc. SIGGRAPH'90), 24 (4), pp.105-114.

Sugihara, K. (1994). A Robust and Consistent Algorithm for Intersecting Convex Polyhedra, Computer Graphics Forum (EUROGRAPHICS '94), 13 (3), pp.C45C54.

Sugihara, K. and Iri, M. (1989). A solid modeling system free from topological inconsistency, Journal of Information Processing, 12 (4), pp.380-393.

Sugihara, K. and Iri, M. (1992). Construction of the Voronoi diagram for "one million" generators in single-precision arithmetic, Proc. IEEE, 80, pp.1471-1484.

Zhu, X., Fang, S. and Brüderlin, B.D. (1993). Obtaining robust Boolean set operations for manifold solids by avoiding and eliminating redundancy, Proc. 2nd ACM Symposium on Solid Modeling, pp.147-154. 